\title{
Tribological Performance Improvement of Bearing Steel GCr15 by an Alternating Magnetic Treatment
}

\author{
Yan-Li Song $^{1,2} \cdot$ Cheng $\mathrm{Yu}^{1,2} \cdot$ Xia Miao $^{3} \cdot$ Xing-Hui Han ${ }^{1,2} \cdot$ Dong-Sheng Qian $^{3} \cdot$ Xu Chen $^{1,2}$
}

Received: 30 December 2016/Revised: 13 March 2017/Published online: 8 May 2017

(C) The Chinese Society for Metals and Springer-Verlag Berlin Heidelberg 2017

\begin{abstract}
This paper discusses the tribological performance of the bearing steel GCr15 treated by an alternating magnetic field. The wear test results showed that the average of wear mass losses decreased by nearly $80 \%$ after the magnetic treatment, compared to those before the magnetic treatment. The micro-hardness and microstructures (i.e., grain size, carbide morphology and dislocation distribution) before and after the magnetic treatment were experimentally investigated, and the mechanism of the tribological performance improvement of the bearing steel GCr15 due to the magnetic treatment was then revealed based on the above results.
\end{abstract}

KEY WORDS: Tribological performance; Magnetic treatment; Bearing steel

\section{Introduction}

Magnetic treatment is a novel method of treating materials placed inside a specific magnetic field at the ambient temperature. By using this approach, at least three main advantages, including potential applications, ease of operation and lack of additive distortions for treated materials, can be found.

In recent years, many facts have proved that a specific magnetic treatment effectively influences solid properties,

Available online at http://link.springer.com/journal/40195.

Yan-Li Song

ylsong@whut.edu.cn

$1 \quad$ Hubei Key Laboratory of Advanced Technology of Automotive Parts, Wuhan University of Technology, Wuhan 430070, China

2 Hubei Collaborative Innovation Center for Automotive Components Technology, Wuhan University of Technology, Wuhan 430070, China

3 School of Materials Science and Engineering, Wuhan University of Technology, Wuhan 430070, China such as changing phase transformation [1, 2], releasing residual stress [3-7], improving fatigue life [8-10], reducing of stamping springback [11] and increasing plasticity [12-14]. As for the alteration of friction and wear performances under a magnetic treatment, only a few reports have been published. Bataineh et al. [15] found that a pulsed magnetic treatment can produce a drill life increase of $35.5 \%$ in tests with aggressive cutting conditions and a drill wear decrease of $10.0 \%$ in tests with mild cutting conditions. Bockstedt et al. [16] pointed out that an imposed magnetic field may be useful for controlling nonlinear processes operating near qualitative changes in behavior as in the mild-to-severe wear transition in tribological processes based on the influences of pulsed magnetic field on thrust bearing washer hardness. Stolarski and Makida [17] found that a horizontal magnetic field causes a temporary reduction in material's hardness and facilitates the removal of wear particles from the contact zone. Yetim et al. [18] observed that samples subjected to the magnetic field during tests showed better wear resistance and lower friction coefficient values than the others. Wei et al. [19] claimed that a DC (direct current) steady magnetic field can reduce friction and wear and promote oxidation. 
In the studies mentioned above, the effects of a magnetic treatment on friction and wear performance are ambiguous and the basic mechanism has not been clearly explained yet. Therefore, in this paper the change in the tribological performance of the GCr15 bearing steel by an alternating magnetic treatment was studied and the effect was interpreted based on the alteration of the mechanical properties and microstructures of the material.

\section{Materials Properties}

In this study, the widely used bearing steel GCr15 (Chinese standard), in a spheroidizing annealing state, was investigated. The elemental chemical composition (in weight percentage) of this material is: $\mathrm{C} 0.95-1.05, \mathrm{Mn} 0.20-0.40$, $\mathrm{Si} \quad 0.15-0.35, \quad \mathrm{~S} \leq 0.020, \quad \mathrm{P} \leq 0.027, \quad \mathrm{Cr} \quad 1.30-1.65$, $\mathrm{Mo} \leq 0.10$, $\mathrm{Ni} \leq 0.30$ and $\mathrm{Cu} \leq 0.25$. The microstructures of GCr15, typically consisting of a soft ferrite phase with a dispersed hard phase composed of spheroidized carbide, significantly affect the service performances of the bearings.

\subsection{Mechanical Properties}

Uni-axial compressive tests were carried out on $\mathrm{GCr} 15$ cylinder specimens with dimensions of $\Phi 8 \mathrm{~mm} \times 12 \mathrm{~mm}$ according to the China National Standard GB/T 7314-2005 [20]. The initial load was set to $1 \mathrm{kN}$, and the final deformation amount of the sample was $20 \%$ of the original height. After the compressive test, the average diameter was about $8.94 \mathrm{~mm}$. The measured mechanical properties are shown in Fig. 1. In particular, the elastic modulus, yield strength and proportional limit of this material obtained by the stress-strain curves are about $210 \mathrm{GPa}, 300$ and $150 \mathrm{MPa}$, respectively.

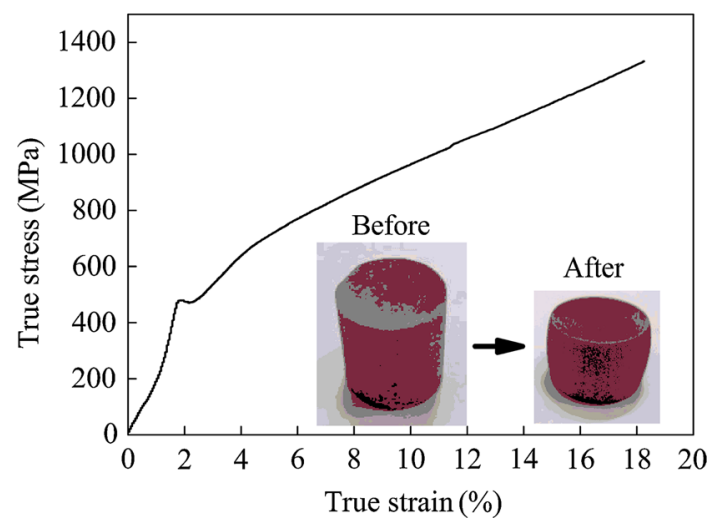

Fig. 1 Uniaxial compressive tests performed on GCr15 steel

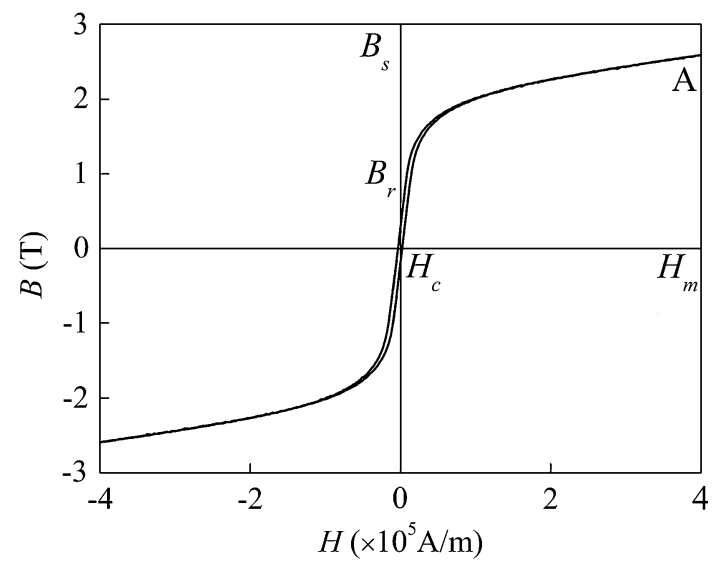

Fig. 2 Hysteresis loop of GCr15 steel [21]

\subsection{Magnetic Properties}

The hysteresis loop of the investigated material is shown in Fig. 2. As can be seen, during the magnetization course (the first quadrant of the coordinate system), the magnetic field strength $H$ gradually increases with the increase in magnetic flux intensity $B$, until the magnetic flux intensity reaches saturation $\left(B_{\mathrm{s}} \approx 2.6 \mathrm{~T}\right)$ at the magnetic field strength $H_{\mathrm{m}}$ of about $4 \times 10^{5} \mathrm{~A} / \mathrm{m}$. $B_{\mathrm{r}}$, at point $H=0$, is the residual magnetic flux intensity, and $H_{\mathrm{c}}$, at point $B=0$, is the coercive force in the hysteresis loop in Fig. 2. The purpose of measuring the hysteresis loop of GCr15 is to determine the magnetic flux density, which can be used in the subsequent magnetic treatment and demagnetization process.

\section{Magnetic Treatment}

The magnetic device basically includes a magnetizator and an AC (alternating current) variable frequency power supply. By using this device, a magnetic field with a maximum magnetic induction of several Tesla and a frequency ranging up to $300 \mathrm{Hertz}$ can be produced in a ferromagnetic material. The magnetic treatment parameters applied here, set as constants, consisted of series of alternating magnetic pulses with a magnetic induction of about 2.0-3.0 T, frequency not higher than power frequency and a treatment time of about several minutes.

\section{Wear Tests and Results}

Considering that the inner and outer rings and the rollers are often made of the GCr15 bearing steel, the materials of both the pin and disk in this test were made of GCr15 in the spheroidizing annealing state. The lubricated pin-on-disk 
sliding tests were carried out by a MM-W1 Friction and Wear Tester at room temperature. The normal load was of $0.1 \mathrm{kN}$. The rotating spindle speed was $400 \mathrm{rpm}$. The lubricating medium was heavy duty gear oil containing emery abrasive grains of 1000 mesh. The ratio of emery to oil was $1 \%$ in mass. The wear mechanism was mainly abrasive wear. In other words, the emery abrasive was pressed into the surface by the normal load, and the surface was then sheared, plowed and cut with the groove grinding marks generated by the furrow action in the sliding process.

The wear tests were conducted in the condition mentioned above, and the test time was set as 480 and $400 \mathrm{~min}$. The sample, shown in Fig. 3, was divided into 4 groups: Groups 1 and 2 were the non-treated samples, while Groups 3 and 4 were the magnetic-treated ones. An analytical balance with the accuracy of $0.0001 \mathrm{~g}$ was then used to measure the wear mass losses of the pin ball.

Table 1 shows the results of the wear tests before and after the magnetic treatments. The averages of wear mass losses of magnetic-treated samples and of the non-treated samples are equal to 0.0104 and $0.0518 \mathrm{~g}$, respectively. The reduction in the average of wear mass losses is by $79.92 \%$ caused by the magnetic treatment, which reveals that the magnetic treatment can remarkably improve the wear resistance.

\section{Discussion}

\subsection{Friction Coefficient Tests}

The surface state of the material has an important influence on the wear performance. The difference between the worn surfaces of GCr15 before and after the magnetic treatments can be deduced from changes in the friction coefficient, which is shown in Fig. 4. In order to obtain reliable results
Table 1 Results of wear tests

\begin{tabular}{llllll}
\hline Group no. & \multicolumn{2}{c}{ Before treatment } & & \multicolumn{2}{c}{ After treatment } \\
\cline { 2 - 3 } & 1 & 2 & & 3 & 4 \\
\hline Time (min) & 480 & 400 & & 480 & 400 \\
Mass before wear (g) & 6.5280 & 6.5330 & & 6.5079 & 6.5263 \\
Mass after wear (g) & 6.4458 & 6.5116 & & 6.4938 & 6.5196 \\
Wear mass loss (g) & 0.0822 & 0.0214 & & 0.0141 & 0.0067 \\
Average of wear mass loss (g) & 0.0518 & 0.0104 & & \\
\hline
\end{tabular}

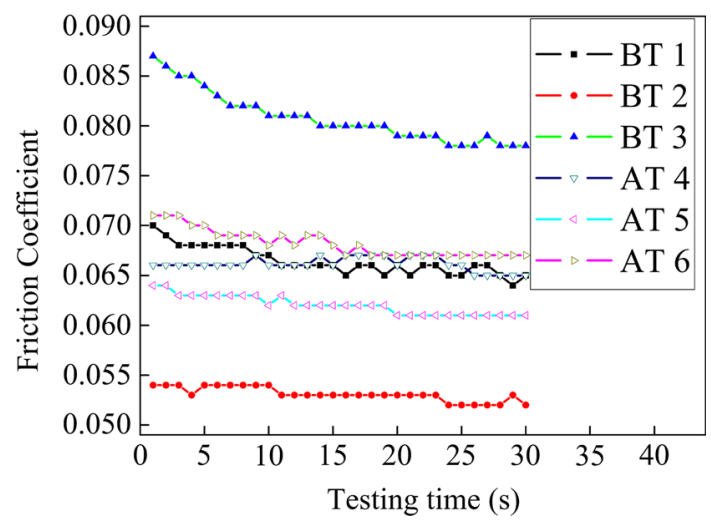

Fig. 4 Measured friction coefficients before and after the magnetic treatments

and reduce occasional errors, the friction coefficient tests of each group were repeated three times.

The results show that the friction coefficients have an evident change in the first $15 \mathrm{~min}$. After that, the friction coefficients reach a stable state. The average and standard deviations of the friction coefficients are shown in Fig. 5. The average friction coefficients remained almost unchanged after the magnetic treatment $(<2 \%)$, while the standard deviation of friction coefficients decreased by
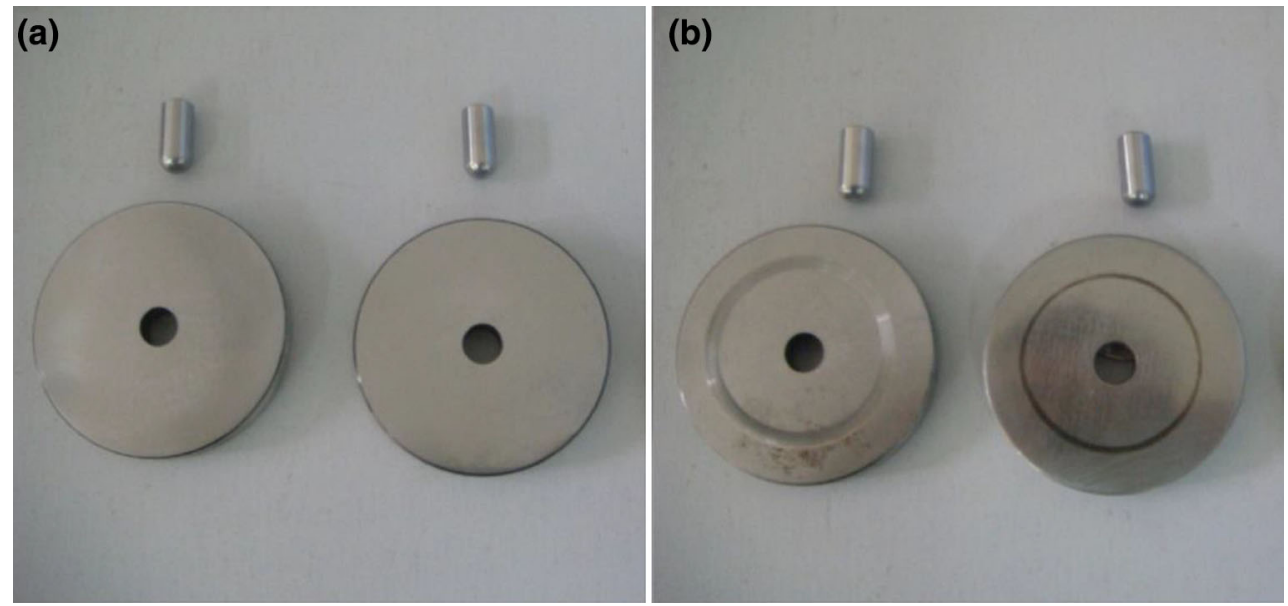

Fig. 3 Photographs of samples before and after wearing tests: a before and $\mathbf{b}$ after treatments 


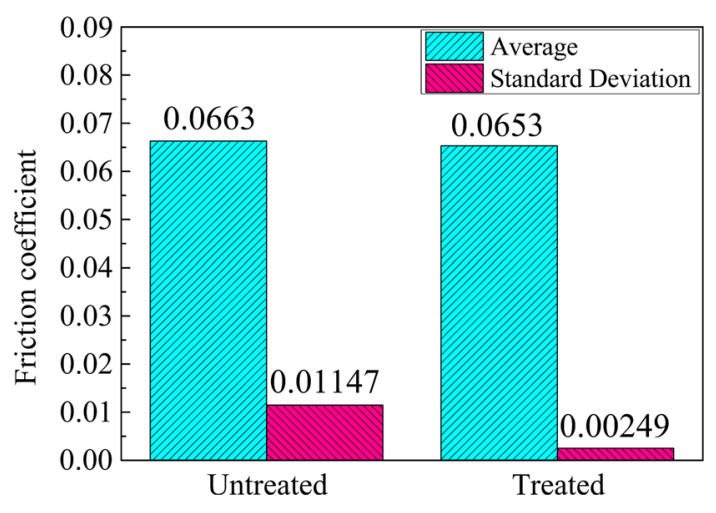

Fig. 5 Average and standard deviation of the friction coefficients

$78.25 \%$ (from 0.01147 to 0.00249 ), indicating that the performance of each sample tends to homogenization by the magnetic treatment.

\subsection{Hardness Tests}

Hardness is a measure of how resistant a solid material is to different kinds of permanent shape change under the action of a compressive force. Generally, the tribological performance of a material improves with the increase in its hardness.

In this work, the micro-hardness of GCr15 samples before and after the magnetic treatments was measured by the HV-1000A micro-hardness tester. The tests were carried out with a load of $100 \mathrm{~g}$ and an holding time of $5 \mathrm{~s}$. Six points in each sample before (or after) the magnetic treatment were randomly selected for the micro-hardness measurement. The measured micro-Vickers-hardness data and their average values are shown in Fig. 6.

The results show that the average hardness of the material arises from 194.8 $\mathrm{HV}$ to $218.1 \mathrm{HV}$, causing an increase of $12 \%$. Meanwhile, the standard deviation of micro-hardness decreases from $7.1 \%$ to $6.4 \%$. Thus, it can

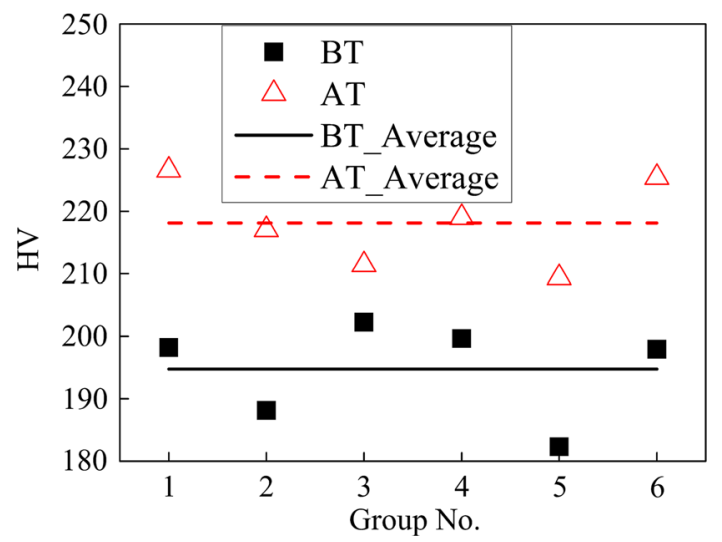

Fig. 6 Micro-Vickers-hardness of GCr15 before and after the magnetic treatments be concluded that the magnetic treatment improves the hardness of the GCr15 steel and makes it more uniform. A higher and more uniform hardness can improve the tribological performance of the metals, which is consistent with the results of the friction and wear tests.

\subsection{Metallographic Structures}

In order to compare the microstructures of GCr15 before and after the magnetic treatments, metallographic tests were carried out by Zeiss Axio Scope A1 Microscope. A corrosive liquid was specially prepared to etch the metallographic samples before the test. Picric acid was added to $40 \mathrm{~mL}$ water, which were heated to $95^{\circ} \mathrm{C}$, until reaching saturation, finally adding two drops of hydrochloric acid. After that, the samples were clipped and put into the above corrosive solution for about $15 \mathrm{~s}$ to etch the grain boundaries.

In order to observe the microstructures in the same region of a same sample, two imprints were marked before the magnetic treatment by using the HV-1000A microhardness tester and were kept for the following metallographic structures tests (Fig. 7).

As shown in Fig. 7, the GCr15 steel in the spheroidizing annealing condition consists of pearlite and cementite and the particles of carbide show a dispersive distribution. There is scarcely any change in grain size before and after the magnetic treatments, different from the research of $\mathrm{Ma}$ et al. [22]. In Ref. [22], the material used was the M42 steel with a grain size of about $40 \mu \mathrm{m}$, and the authors attribute the refinement of the austenite grain to the segmentation effect of acicular martensite precipitation. However, the GCr15 steel has a quite different metallographic structure, compared to the M42 steel. The grain size of the GCr15 steel is rather small (about $10 \mu \mathrm{m}$ ) at room temperature, and the precipitation of a hardened phase by the magnetic treatment is not evident. Therefore, the segmentation action to grains is not clear distinct, so that the improvement in hardness and tribological performance seems to be little related to the grain size in this work.

\subsection{Carbide Distribution}

The carbide distribution on the pearlite matrix of the highcarbon bearing steel GCr15, shown in Fig. 8, was observed by SEM. Figure 8 a shows that the carbide particles distributed on the pearlite matrix in the annealed GCr15 steel. The common way of the carbide particles growth is the original position nucleation in the spheroidizing annealing process of the pearlite structure. Also, the carbide particles of large size are majority, compared to those of small size. In other words, the distribution of the carbide particles is non-uniform before the magnetic treatment. However, the 

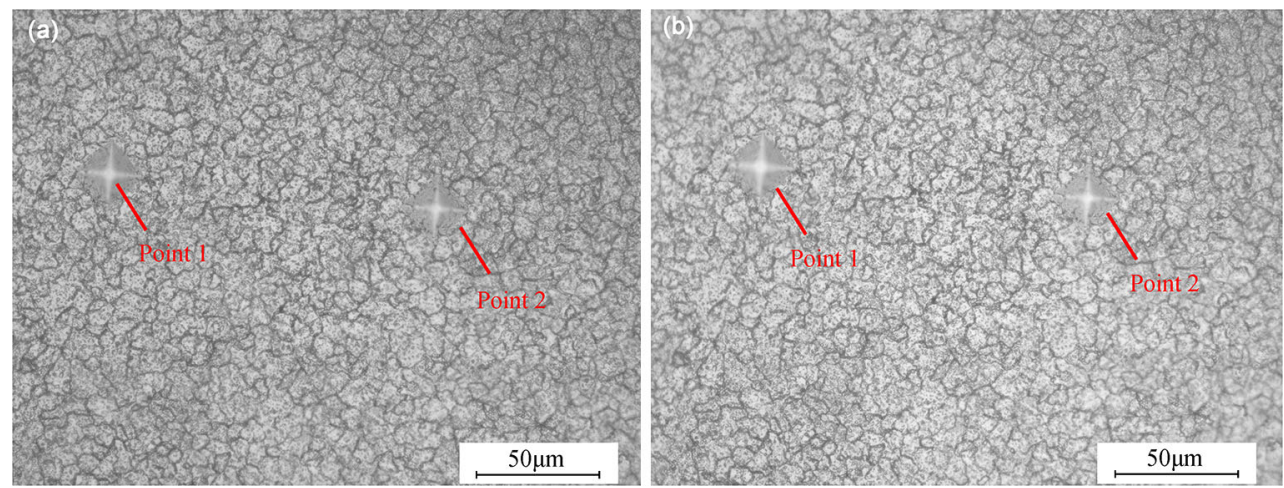

Fig. 7 Metallographic images of GCr15 before $\mathbf{a}$ and after $\mathbf{b}$ the magnetic treatments
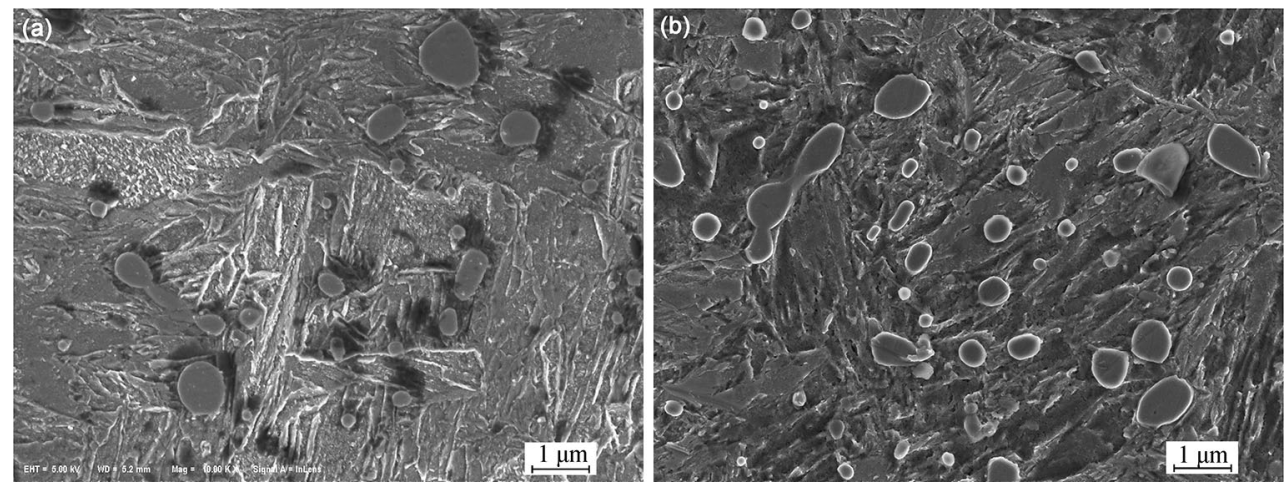

Fig. 8 Carbide distribution of GCr15 before and after the magnetic treatment: a before treatment; $\mathbf{b}$ after treatment

larger carbide particles become finer and more uniform after the magnetic treatment, although there is still a small fraction of carbide particles of large size, as shown in Fig. 8b.

A statistical analysis of the carbide particles size by using Image-Pro Plus (Fig. 9) shows that the carbide particles become finer, with an average diameter decreasing from 0.45031 to $0.31371 \mu \mathrm{m}$, causing a reduction of nearly $30 \%$. Therefore, the refinement of the carbide particles due to the magnetic treatment promotes their decomposition.

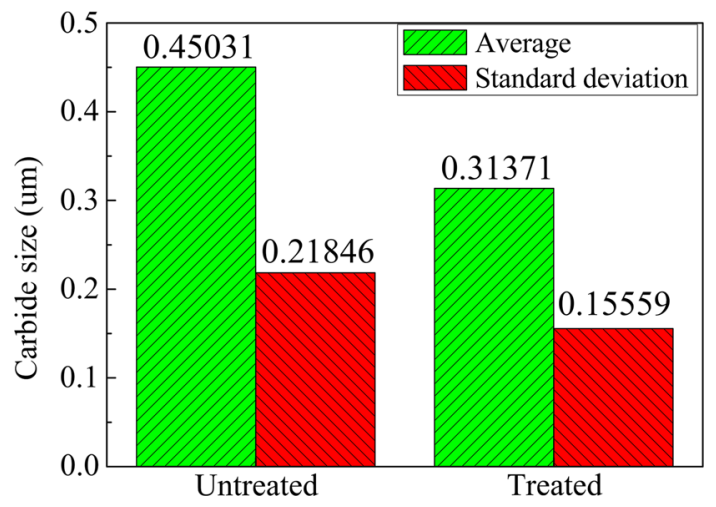

Fig. 9 Carbide size of $\mathrm{GCr} 15$ before and after the magnetic treatments
The reasons for the carbide particles refinement may be the interaction force generated by the magnetic field. Due to the different ferromagnetic properties of the carbide and the pearlite matrix in $\mathrm{GCr} 15$, the forces acting on them under a magnetic field are different. Thus, interactions between the carbide and the pearlite matrix are generated during the magnetic treatment. The alternating magnetic field generates micro-regions of plastic deformation which produce deformations of the carbide particles and the pearlite matrix. If the deformation amount exceeds a critical value, cracks appear on the carbide particles due to its lower ductility, compared to other soft phases. The filling of the other soft phases into the cracks contributes to a further decomposition of carbide particles, as shown in Fig. 10. Therefore, the carbide particles become finer and distributed more uniformly after the magnetic treatment. The fine and uniformly distributed carbide particles have a positive effect on the wear resistance [23].

\subsection{Dislocation Distribution}

Generally, the increased ability to resist plastic deformation can improve the wear resistance [24], while the plastic deformation is carried out through the movement of the dislocations. Thus, it is significant to investigate the 
(a)

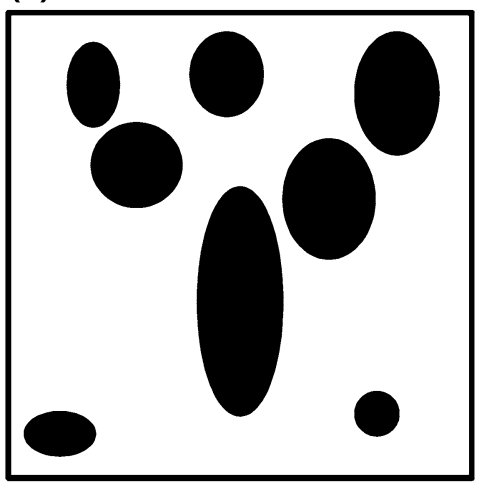

(b)

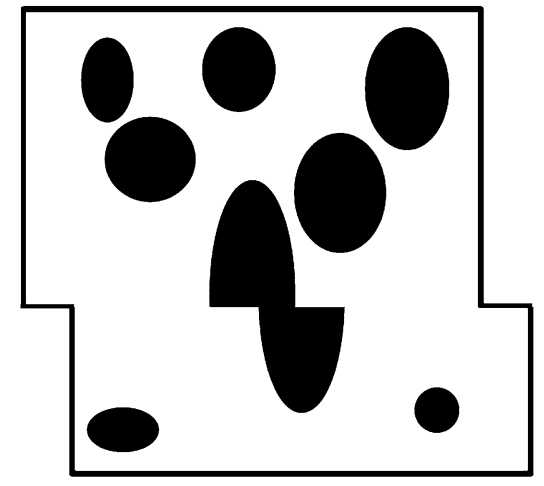

(c)

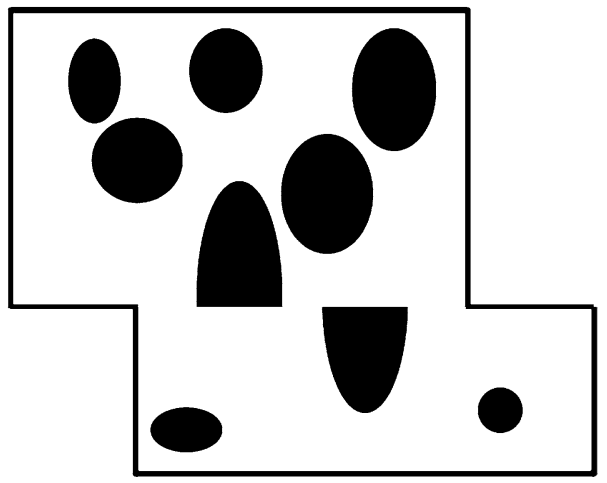

Fig. 10 Process of carbide particle refinement under the action of a magnetic field: a the initial state, $\mathbf{b}$ the intermediate state and $\mathbf{c}$ the final state

dislocation distribution before and after the magnetic treatments. The preparation process of the film sample used in the transmission electron microscope (TEM) experiment is briefly described below. Firstly, the intermediate region of sample was made into a film with a size of $8 \mathrm{~mm} \times 4 \mathrm{~mm} \times 0.4 \mathrm{~mm}$ by wire cut electrical discharge machining (WEDM). The sample was then worn thin to 60-80 $\mu \mathrm{m}$ on 1000 mesh metallographic sandpaper in order to remove the surface damage caused by WEDM. Then, the film sample was punched into a small wafer with a diameter of $3 \mathrm{~mm}$ and thinned into a bowl-shaped pit. Finally, the sample was thinned by using an ion beam thinner with the purpose of increasing the thin area and removing the contamination on the surface.

Figure 11 shows the dislocation structures of the samples before and after the magnetic treatment under the TEM. Since the GCr15 steel used here is in a spheroidizing annealing condition, there is no large plastic deformation inside the material. Hence, the dislocation density is at a low level and the dislocations are displayed more clearly before the magnetic treatment (Fig. 11a). Conversely, the dislocation density of the sample after the magnetic treatment increases significantly and the dislocations are distributed more uniformly in the entire field of view, despite dislocation lines intersecting and bending in some local areas (Fig. 11b).

The microscopic mechanism of the increase in the dislocation density caused by the magnetic treatment can be explained by the mechanism of Frank-Read dislocation multiplication. The driving force $\tau$ of the dislocation multiplication is the shear stress component of the elastic stress on the slip surface, which is consistent with the direction of Burgers Vector $\boldsymbol{b}$ and is mainly provided by the force of the magnetic field. This force is given by [25]

$\tau=\mu_{0} M_{\mathrm{s}} H \cos \theta$,

where $\mu_{0}$ is the permeability of vacuum, $M_{\mathrm{s}}$ is the saturation magnetization, $H$ is the magnetic field intensity, and $\theta$ is the angle between the magnetic field and the direction of magnetization.
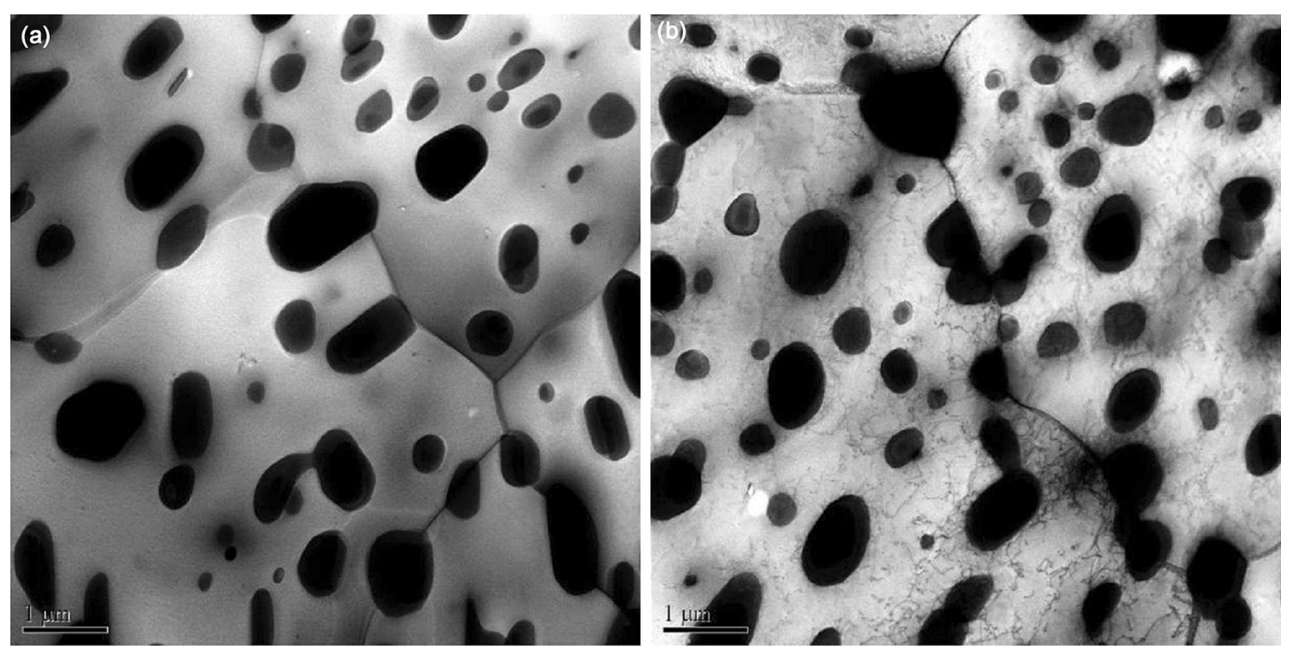

Fig. 11 Dislocation structures before $\mathbf{a}$ and after $\mathbf{b}$ the magnetic treatments 
The magnetic force changes over time under the effect of an alternating magnetic field. The restoring force $\sigma$ of the dislocation line tension is given by [26]

$\sigma=\frac{G b}{L}$,

where $G$ is the shear modulus, $b$ is the Burgers Vector, and $L$ is the length of dislocation line. When the magnetic force $\tau$ is greater than the restoring force $\sigma$ of the dislocation line tension (i.e., $\tau>\sigma$ ), the dislocation line, two ends of which are coiled to form a complete dislocation loop (Fig. 12), becomes unstable and begins to move. According to Eqs. (1) and (2), the critical magnetic field intensity can be expressed by

$H=\frac{k}{L \cos \theta}$,

where $k$ is a constant related to the material and is given by

$k=\frac{G b}{\mu_{0} M_{\mathrm{s}}}$.

As shown in Fig. 12, dislocation loops are produced repeatedly and endlessly in the order of a, b, c, d and a under the effect of the alternating magnetic field, which results in a dislocation multiplication.

Multiplicative dislocation loops, in a state of high energy and instability, are decomposed into a plurality of dislocation lines with smaller Burgers Vectors. Meanwhile, the dislocations with the opposite Burgers Vectors disappear once they meet, while some other dislocations intersect and form dislocation networks or dislocation walls. When the stacking fault energy reaches a critical level under the further action of the applied magnetic field, the dislocation walls become connected with each other to form sub-grain boundaries (Fig. 13).

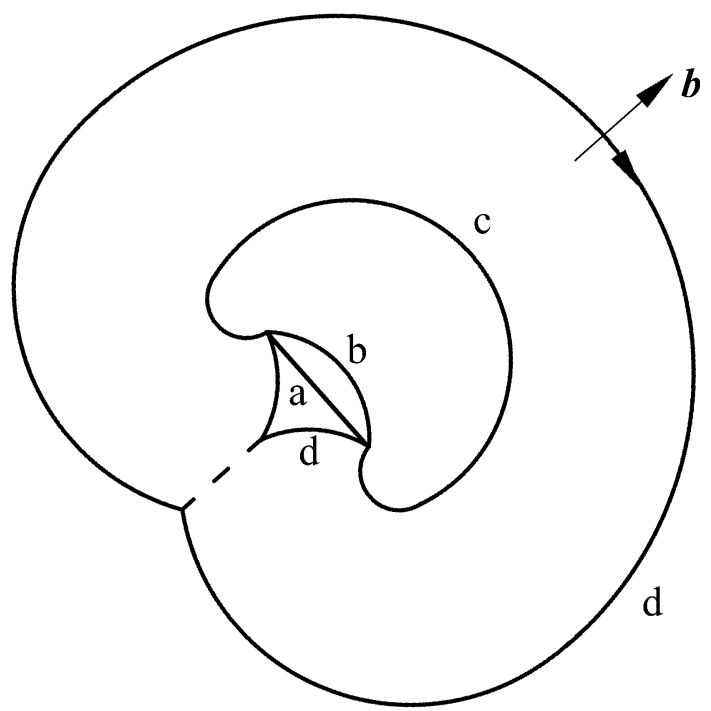

Fig. 12 Mechanism of Frank-Read dislocation multiplication [27]

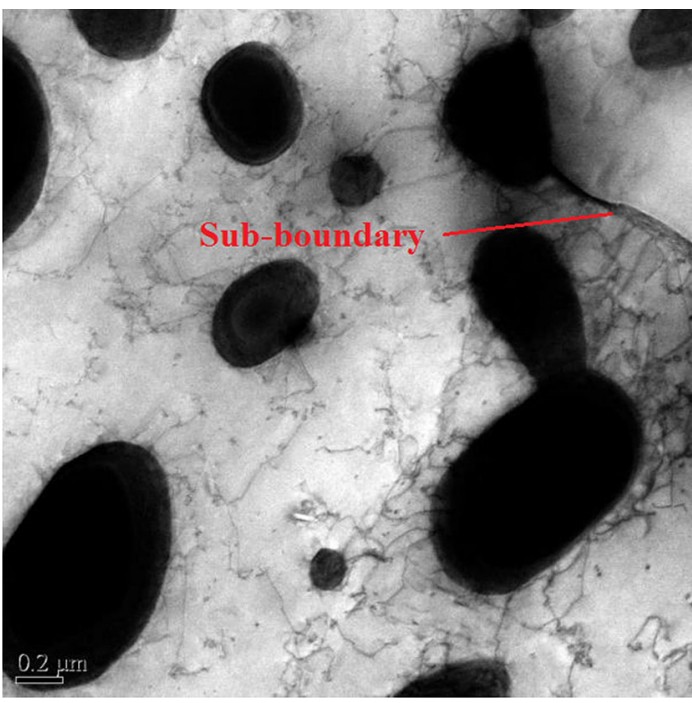

Fig. 13 Cellular sub-grain structure after magnetic treatment

The formation of dislocation walls and sub-grain boundaries under the action of the magnetic treatment indicates a dislocation strengthening. In turn, this causes an increase in the plastic deformation resistance of the material and finally improves its wear resistance.

\section{Conclusions}

1. After the magnetic treatment, the average wear mass losses were reduced by $79.92 \%$, while the average friction coefficients remained unchanged $(<2 \%)$ with a reduction of $78.25 \%$ in the standard deviation. Besides, the average hardness increased by about $12 \%$ with a small fluctuation in the standard deviation.

2. After the magnetic treatment, the carbide particles became finer and the dislocation density increased with the formation of dislocation walls and sub-grain boundaries. The critical condition equations for the dislocation multiplication under the action of magnetic field were established.

3. The improvement in the wear performance of $\mathrm{GCr} 15$ is due to a higher and more uniform hardness, basically caused by a more homogeneous distribution of the carbide particles and of the dislocations after the magnetic treatment.

Acknowledgements This project is supported by the National Natural Science Foundation of China (Grant Nos. 51305317 and 51675392), the Natural Science Foundation of Hubei Province of China (Grant No. 2014CFA119) and the Special Project of Technological Innovation of Hubei Province (Grant No. 2016AAA053).

We also show our thanks to the MatProFuture Project. 


\section{References}

[1] C.C. Koch, Mat. Sci. Eng. A 287, 213 (2000)

[2] C. Gheorghies, I.I. Stefanescu, J. Iron. Steel Res. Int. 17, 45 (2010)

[3] B.E. Klamecki, J. Mater. Process. Technol. 141, 385 (2003)

[4] Y.L. Song, L. Hua, B. Wang, J. Wuhan Univ. Technol. 24, 857 (2009)

[5] Z.P. Cai, X.Q. Huang, Mat. Sci. Eng. A. 528, 6287 (2011)

[6] Y.L. Song, L. Hua, J. Mater. Sci. Technol. 28, 803 (2012)

[7] S. Wu, A. Lu, H. Zhao, H. Fang, F. Tang, J. Mater. Process. Technol. 132, 198 (2003)

[8] Y. Fahmy, T. Hare, R. Took, Scr. Mater. 38, 1355 (1998)

[9] A. Celik, A.F. Yetim, A. Alsaran, Mater. Des. 26, 700 (2005)

[10] B.T. Lü, S.R. Qiao, X.Y. Sun, Scr. Mater. 40, 767 (1999)

[11] E. Iriondo, M.A. Gutiérrez, B. González, J.L. Alcarazb, G.S. Daehn, J. Mater. Process. Technol. 211, 909 (2011)

[12] Y.I. Golovin, Phys. Solid State 46, 789 (2004)

[13] V. Fleurov, M. Molotskii, Phys. Rev. Lett. 78, 2779 (1997)

[14] M.I. Molotskii, Mat. Sci. Eng. A 287, 248 (2000)
[15] O. Bataineh, B. Klamecki, B.G. Koepke, J. Mater. Process. Technol. 134, 190 (2003)

[16] J. Bockstedt, B.E. Klamecki, Wear 262, 1086 (2007)

[17] T.A. Stolarski, Y. Makida, Wear 271, 1109 (2011)

[18] A.F. Yetim, H. Kovaci, M. Aslan, A. Çelik, Wear 301, 636 (2013)

[19] Y.H. Wei, Y.Z. Zhang, Y. Chen, S.M. Du, Tribol. Int. 57, 162 (2013)

[20] GB/T 7314-2005, Metallic Materials-Compression Testing at Ambient Temperature, China National Standard, 2005

[21] X. Miao, D.S. Qian, Y.L. Song, J. Mech. Eng. 50, 112 (2014)

[22] L.P. Ma, Z.Q. Liang, X.B. Wang, W.X. Zhao, L. Jiao, Z.B. Liu, Acta Metall. Sin. (Engl. Lett.) 63, 137 (2015). (in Chinese)

[23] C.Q. Gao, Heat Treat. 8, 62 (1987)

[24] G.M. Sorokin, V.N. Malyshev, Tribol. Int. 41, 515 (2008)

[25] P.J. Ferreira, J.B.V. Sande, Scr. Mater. 41, 117 (1999)

[26] L.P. Ma, W.X. Zhao, Z.Q. Liang, X.B. Wang, L.J. Xie, L. Jiao, T.F. Zhou, Mater. Sci. Eng. A 609, 16 (2014)

[27] S.H. Yang, Crystal Dislocation Theory Foundation (Science Press, Beijing, 1988), pp. 46-54 